\title{
A Suggested Design for Head Cover from Smart Tissue for women with Multiple Sclerosis in Saudi Arabia
}

\author{
Dr. Elham Fathy Abdel Aziz and Doaa Khalaf Abdul Rahman Al-Maliki \\ Associate Professor, Department of Fashion and Textile, College of Human Sciences and Design, King \\ Abdulaziz University, Master's student in the Department of Fashion and Textile, College of Human Sciences \\ and Design, King Abdulaziz University
}

\begin{abstract}
Multiple sclerosis is known as one of the most common neurological and chronic diseases, and it usually affects young people and adults (20-40 years old). And it is a disease that affects the central system and its causes are unclear, but hypotheses suggest that it is due to a defect in the immune system. Many studies have confirmed that there is a close relationship between clothing and improving the quality of general health for the human being. The research aims to reach design proposals for headscarf to protect the head, shoulders, spine and eyes, which would contribute to improving the lives of women with multiple sclerosis.
\end{abstract}

Keywords: Multiple sclerosis, KSA, Head Cover from Smart Tissue

DOI: $10.7176 / \mathrm{ADS} / 91-07$

Publication date: March 31st 2021

\section{Introduction}

Multiple sclerosis is known as one of the most common neurological and chronic diseases, and it usually affects young people and adults (20-40 years old). And it is a disease that affects the central system and its causes are unclear, but hypotheses suggest that it is due to a defect in the immune system (Ghazali, 2018). And it is estimated that 2.3 million people have multiple sclerosis worldwide, based on the latest statistics of the Ministry of Health website 2018. The statistics also indicate that the disease affects women more than men, with a ratio of 1 to 3 (Abyan, 2013). The Ministry also invited people with multiple sclerosis to join support groups to get to know people of similar health status. And it recommended that people with multiple sclerosis avoid exposure to high heat as much as possible, such as: the sun's heat, or bathing in hot water, or exposure to hot air, and from here we find the importance of research.

Many studies have confirmed that there is a close relationship between clothing and improving the quality of general health for the human being (Ahmad, 2010). Therefore, smart textiles appear to us, which have contributed to solving many research problems, and are defined as tissues formed by the integration between textiles and electronic or physical components to provide improved functions (Chapman, 2013). The use of smart fabrics has been popular since the late 1980's, via science fiction films. And the idea that movie characters should be able to take advantage of technology to protect the body, in the form of wearable computers and these fabrics, their development was not limited to the development of the concept of computers. Rather, it went beyond that as various technologies supported by the United Nations, which were not expected to spread rapidly within a few years, such as protective clothing from any kind of risk (Al-Ani, 2017).

The research aims to reach design proposals for headscarf to protect the head, shoulders, spine and eyes, 
which would contribute to improving the lives of women with multiple sclerosis. Made of smart fabric that contains porous polyethylene nanofibers to shield from the sun's infrared rays. Polyethylene fabric is known as one of the synthetic fibres that fall under the polyolefin's family. And with the scientific progress in the sixties of the last century, its use was no longer limited to a few industrial applications, rather, we find them in the fibres for the manufacture of monofilament yarns, and they have several uses (Gad, 2010).

The importance of the research revolves around access to cooling the head of people with Multiple sclerosis while they are under the sun in hot environments so that it is self-cooling and without sweating. And this is confirmed by the study (Yi Cui, 2016) that the introduction of a nanopore polyethylene compound helps people relieve heat stress and cool their bodies by using clothes instead of using air conditioners. But even lightweight cheesecloth clothes absorb the infrared rays our bodies give off and trap heat. Yi Cui's study found that fibres called nanopore polyethylene - which are used in lithium-ion batteries - let radiation escape away from the body (Al Nahass, 2014). And unlike advanced sportswear, which relies on sweating to cool the wearer, nanopore polyethylene works without the need for sweating, and compared to pure cotton, cotton allows only $1.5 \%$ of infrared waves to pass through, whereas, polyethylene allows $96 \%$ of infrared waves to be radiated from our skin. The research problem appears in the increase in the number of people with Multiple sclerosis in the Gulf region, reaching 11,350 male and female patients (Al-Jaishy, 2012).

Based on the initiatives of the National Transformation Program 2030 in the Kingdom of Saudi Arabia, the Authority for the Care of Persons with Disabilities - established by a decision of the Council of Ministers in $1439 \mathrm{AH}$ - sought to increase the participation of people with Multiple sclerosis in the family and society, and overcoming the challenges that face them, as well as harnessing all capabilities to serve them, since the number of women affected by this disease more than men, and based on the nature of the disease that focuses on the youth group, the research linked the problem of young Saudi women with this disease to their exit in the hot climates that overwhelm most regions of the Kingdom. Where the research studies the possibility of making designs for headscarves for women with Multiple sclerosis that are appropriate in terms of aesthetic and functional aspects. The headscarf of Muslim women is characterized as everything that is covered by a dress and other thing (Tashkandi, 2014). It may be called the shirt, the khimar, the robe, and the veil, and it is a wide dress for women that is wider than the robe, and by which women cover their head, chest and back (Halali, 2005).

\section{Research questions}

1. What is the possibility of using smart fabric to make a headscarf to reduce the feeling of heat and without sweating, suitable for people with Multiple sclerosis in hot areas?

2. What is the possibility of proposing aesthetic and functional designs for a headscarf suitable for women with Multiple sclerosis in hot regions?

\section{Research objectives}

- $\quad$ The use of smart fabric to make a headscarf to reduce the feeling of heat and without sweating suitable for women with Multiple sclerosis in hot areas.

To suggest aesthetic and functional designs for a headscarf that fits the needs of women with Multiple sclerosis in hot regions. 


\section{Research importance}

- $\quad$ The research contributes to solving social and health issues by merging histology and other sciences in terms of selecting the appropriate and improving the properties of some fibres to make them adapt to new conditions and problems.

Providing means of comfort for many of those with immune diseases to increase the participation of infected persons in the family and society, and to overcome the challenges facing them.

\section{Research Methodology}

The research follows the descriptive approach (the analytical study - the applied study) so that it helps in analysing the basic elements that make up the subject of the study followed, which is the work of suggested designs for a headscarf for women with Multiple sclerosis. To prevent high body temperature while they are in the sun, through the use of the systematic tools of analytical description.

\section{Research limits}

\section{Objective limits}

The search was limited to headscarves for veiled women (scarves): fabrics treated with nano-porous polyethylene.

As for the programs used for designs: The Illustrator program, the Cloe Design Program.

Spatial limits: Multiple sclerosis patients in hot regions of the Kingdom of Saudi Arabia.

\section{Research hypotheses}

- $\quad$ The use of a smart cloth that lowers the body temperature without sweating for women with Multiple sclerosis while they are in the sun in hot regions.

- $\quad$ There are statistically significant differences between the functional and aesthetic designs of headscarves for women with Multiple sclerosis, and the views of women.

\section{Research tools}

Through the research questions, we find the multiplicity of the tools used in the study:

- Use the questionnaire (for women with Multiple sclerosis) to collect information and data about the design needs of headscarves for women with Multiple sclerosis.

- $\quad$ The use of the questionnaire (for the casualties) to measure the estimate of the sample's acceptance of the various designs in terms of (the functional aspect, the aesthetic aspect).

\section{Terminology}

\section{- $\quad$ Multiple Sclerosis}

It is a neurological disorder, represented in the rupture of the myelin membrane surrounding the cylindrical axis, because the immune system fails to distinguish its own tissues, and attacks the membrane surrounding the cylindrical axon. (Balcer, 2007).

Thompson and others (Thompson, 2000) know it as a case of a fierce attack on the brain for unknown 
reasons that leads to multiple relapses of the human body as a result of the inflammation that occurred due to this attack, especially on the spinal cord, and this attack lasts for 24 hours at least.

\section{- $\quad$ Smart Fabrics}

Fabrics formed by the integration of textiles with electronic components to provide enhanced functionality. Smart textiles are used to monitor the location and physiological condition of individuals, and to protect soldiers in conflict areas (Chapman, 2013). (Al-Ani, 2018) indicates that the concept of smart fabrics means mixing materials, fabrics and electronics that produce fabrics with multiple capabilities, so that they are able to perform in wider fields, (Muhammad, 2019). It is the link between the human body and technology and thus performs our functions - comfort, safety, sports and health - with ease.

\section{Theoretical framework}

The concept of a healthy clothing culture is one of the important concepts that all groups of society should be familiar with because of the importance of raising awareness of clothing towards what we choose for ourselves and for our children, whereas, clothes have an effect on the health of the individual, as well as on the environment surrounding a person, which affects his health (Ahmed, 2010). With the advancement of technology and its entry into the world of the textile industry, including the production of reactive textiles, it has become an important focus in human life as it poses a challenge to humans, due to the climatic conditions prevailing in the Kingdom of Saudi Arabia, this has led to the desire to use modified (smart) textile materials in the design that are compatible with the nature of the climate (Al-Ani, 207).

\section{Smart fabrics}

The concept of smart fabrics has been popularized since the late 1980s, through science fiction films and the idea that cinematic characters should be able to take advantage of technology to protect the body, in the form of wearable computers and these fabrics, their development was not limited to the development of the concept of computers, but went beyond that as the various technologies supported by the United Nations spread. Which was not expected to spread rapidly within a few years, like protective clothing from any kind of risk (AlAni, 2017).

\section{Smart Textiles}

Fabrics formed by the integration of textiles with electronic components to provide enhanced functionality. Smart textiles are used to monitor the location and physiological state of individuals, and to protect soldiers in conflict areas (Chapman, 2013).

Among the types of smart fabrics are: comfort - safety - sport - health (Mattile, 2006). 


\section{Smart fabrics and improving human life}

Today, smart clothes have become more effective for any external variables, regardless of their accuracy, whether the variable is temperature, light or humidity. And these materials acquired the quality of intelligence because they sense the surrounding conditions and respond in response, hence, smart fibres represent the next generation that sustains people's lives, the clothes may gain us the required warmth and in the required time without the need to change clothes to cope with the environmental change taking place (Al-Jamal, 2004).

\section{Examples of smart tissues include}

Smart fabrics are made of synthetic fibres with special properties that sometimes surpass those of natural fibres, especially the comfort and wear properties, and their durability of treatments and alkalis.

\section{Industrial polyethylene fibres}

Polyethylene fibres are one of the types of synthetic fibres that fall under the polyolefin's family (Gad, 2010). With the scientific progress in the sixties of the last century, their use was no longer limited to a few industrial applications. Rather, we found them in the fibres for the manufacture of single filament yarns, and it has several uses (Yi Cui, 2016). The interest in these fibres for this study is their properties in preventing the transmittance of harmful sun rays, but being rigid and inflexible, they have been treated by specialized scientists by making them nano-pores and increasing the property of elasticity.

\section{Nanotechnology and its application to smart tissues}

Nanotechnology and its applications have revolutionized many industrial fields, which led to attracting the attention of workers in various research fields, it is a promising technology that heralds a huge leap in all branches of science by arranging particles of matter next to each other in a way we cannot imagine and at the lowest possible cost, nanotechnology is considered a modern scientific industrial revolution that all countries of the world are now interested in (Al-Nahass, 2014).

\section{Polyethylene nano-pore fibres}

A toxic nano-polyethylene compound is a component of a common plastic material known as Nano PE, researchers have increased interest in the property that distinguishes it by allowing infrared rays to pass directly through it when it turns into a sticky cover, but the inner glued cover also traps moisture and it is transparent. Therefore, before using them in clothes, researchers had to treat some properties such as elasticity, waste water and light, where they found a nanostructured polyethylene used in the manufacture of batteries is opaque to 
visible light while it remains transparent to infrared, and that means it helped let infrared rays pass more easily too, and then they developed a chemical mixture to treat the plastic that allowed it to pass water, like cotton. This contributes to making the resulting plastic more flexible as a fabric, and the study found the creation of a triple material with two pieces of polyethylene with a cotton net in the middle that contributed to reaching the desired fabric (Yi Cui, 2016).

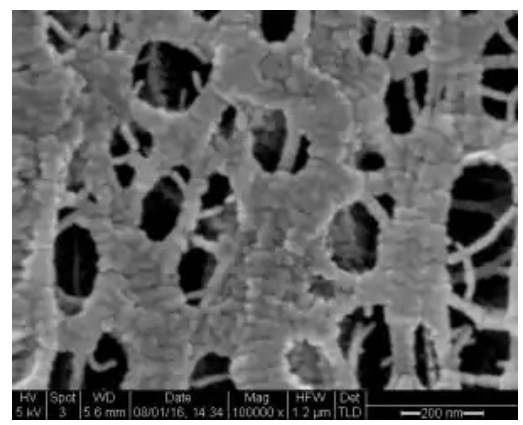

Photo 1 Yi Cui, 2016 Nano PE

\section{Multiple sclerosis and smart fabrics}

The number of people with multiple sclerosis has increased in the Gulf region in particular and the world in general (Al-Jishi, 2012), and based on what was stated in the initiatives of the National Transformation Program 2030 in the Kingdom of Saudi Arabia, the Authority for the Care of Persons with Disabilities - which was established by a decision of the Council of Ministers in $1439 \mathrm{AH}$, sought to increase the participation of people with Multiple sclerosis in the family and society, and overcoming the challenges that face them, as well as harnessing all capabilities to serve them, where it becomes clear to us the importance of optimizing the lives of injured people through smart tissues that contribute to solving some of the difficulties facing this group, including protection from heat and sunlight, using smart and treated cloth.

\section{What is multiple sclerosis}

It is known as a case of fierce attack on the brain for unknown reasons that lead to multiple relapses of the human body as a result of the inflammation that occurred as a result of this attack, especially on the spinal cord, this attack lasts for at least 24 hours (Thompson, 2000). Which in turn provides requirements that the patient must deal with and face in order to alleviate the pressure that results from the disease and achieve balance and psychological comfort in order to maintain his health (Khraifi, 2017).

\section{Multiple sclerosis is affected by climate}

Multiple sclerosis is the most common disabling neurological condition in young adults. A patient's quality of life is greatly influenced by Multiple sclerosis; however, few studies have focused on finding relationships between the multiple sclerosis patient factor with the quality of life, and the goal is to assess the patient's quality of life while measuring a number of influences, including being affected by climate (Algahtani, 2020).

The study indicated that $60-80 \%$ of Multiple sclerosis patients show adverse clinical symptoms when 
their body temperature rises, the need to develop treatment strategies to overcome the problem of thermoregulation (Antonia, 2019).

\section{Previous studies}

Algahtani (2020) conducted a study entitled "Assess the quality of life of care providers for multiple sclerosis patients in the Kingdom of Saudi Arabia". The study indicated that multiple sclerosis is the most disabling neurological condition and the most prevalent among young people. As the patient's quality of life is greatly affected by Multiple sclerosis due to the worsening of symptoms, however, few studies have focused on finding the relationships between the multiple sclerosis patient factor with the quality of life, and the goal is to assess the patient's quality of life with a number of influences (age, gender, marital status, occupation and history of diagnosis).

Khraifieh (2015) conducted a study entitled "The quality of life of patients with multiple sclerosis". Through this study, we learn about the dimensions of the quality of life that patients with multiple sclerosis suffer. This study refers to learning about the psychological life of multiple sclerosis, where the results also showed that both genders enjoy a deteriorating quality of life. These results have been interpreted in light of the results of previous studies in addition to field observations. The reason for the deterioration in the quality of life can be due to several factors, such as the duration of the disease, the type of infection, and the study confirmed that the climate factor affects the emergence of side disorders and accelerates seizures and relapses after the seizure.

\section{Comment on the studies}

The study (Kharaifa, 2015- Algahtani, 2020) is concerned with solving patients' issues in terms of improving life, the two studies found that the climate factor moves in a directional way with the deterioration of the patient's condition. As for the current study, it was concerned with developing solutions and facilities for female patients to cope with the climate situation, in particular from the patients' exposure to infrared radiation while leaving the house by proposing designs for the headscarf made of smart fabric.

\section{Studies using polyethylene material in other fields:}

Slimane (2020) conducted a study entitled "Operation of the woven polyethylene terephthalate fabric with cowpea protein and biopolymer compound: applications to enhance wettability and UV protection properties". In this study a simple strategy was developed to enhance the protection from water and UV rays of the woven surface of polyethylene terephthalate by inoculating green bean protein with modified epoxy of gum Arabic, where the research found improved water resistance and UV protection for knitted fabric, and the study indicated that the UV protection factor rating for modified PET knitted fabric is higher, this indicates that the modified layer caused the scattering and absorption of UV light.

Nga Nguyen (2017) conducted a study entitled "Multifunctional Ti-O coating on a polyethylene terephthalate fabric produced using a pulse magnetron spray system" in which he claimed Practical and comfortable textiles have been widely used for outdoor protective clothing against outside moisture, wind and cold, along with transferring good sweat from inside to outside. Hydrophobic textiles have attracted various applications, such as water resistance, self-cleaning, bioactive properties, while hydrophilic textiles exhibit 
wettability, breathability, wicking and a smooth look. And by spraying the high-energy pulsed magnetron, in this study, on the polyethylene terephthalate fabric, the results indicate that the Ti-O coated PET fabric exhibited a hydrophobic property.

Comment on the studies

The two studies (Nga Nguyen, 2017- Slimane, 2020) confirmed the use of polyethylene material in other areas, and the two studies concluded that a polyethylene terephthalate fabric can be treated during its weaving to resist a number of functions such as sunlight, water contact, the current study differed as it used the nanopore polyethylene material to achieve cooling and equalization of body temperature without sweating under the sun's rays.

\section{Studies that use smart fabrics to cool the body}

Wei Wei (2020) conducted a study entitled "Fabric coated with cellulose acetate for human body cooling". A tissue has been developed that cools the skin in both sunlight and also protects the human body from the extremely hot work environment, by developing a concept that combines solar reflection with "passive radiative cooling" to enhance both the blocking of solar energy and heat dissipation through long-wave infrared radiation, and modified textiles can also avoid actual human skin overheating of 0.6-1 under sunlight.

Song (2020) conducted a study entitled "Polyvinylidene fluoride fabric for passive cooling of human body". PVDF porous polyvinylidene fluoride fibres and woven tissues were manufactured for passive human body cooling by combining nanoscience and molten spinning techniques using the microfiber method, the research found that the improved porous PVDF tissue could be avoided by simulating an increase in the human body temperature of $17.7^{\circ} \mathrm{C}$.

Lijun $\mathrm{Lu}$ a (2020) conducted a study entitled "A proposal for fibre-shaped lithium-ion batteries". The problem lies in the wearable devices and the reason is that they are hard and high density. Meanwhile, the flexible and lightweight planar batteries cannot be fitted to the fabric well due to their poor permeability, which reduces the degree of wearing comfort of the fabric. Therefore, a lithium-ion battery has been used which can solve all the above problems due to its light weight, flexibility, and expandability, but installing them in the fabric will be complicated.

Antonia (2019) conducted a study entitled "The effect of pre-cooling therapy on the physical performance and functional capacity of patients with multiple sclerosis: a systematic review". The study indicated that $60-80 \%$ of Multiple sclerosis patients show opposite clinical symptoms when their body temperature rises, and the need to develop treatment strategies to overcome the problem of thermoregulation, where the previous study tended to discuss the current knowledge of the effects of cryotherapy on the functional ability of patients with multiple sclerosis, where this study reviewed studies that tried active cooling methods, using clothing or other materials that are cooled by circulating the fluid through a tube and the results of all the studies analysed in this study showed that cryotherapy can prevent worsening of symptoms, due to maintaining the body temperature of Multiple sclerosis patients and preventing negative effects. Therefore, such strategies can serve as a complementary treatment approach for patients with multiple sclerosis. 


\section{Comment on previous studies}

Four studies used smart fabrics for body cooling Wei Wei, 2020. Lijun Lu a, 2020. Antonia, 2019. Song, 2020), where the results of the studies showed that cellulose acetate as a material and fabric of polyvinylidene fluoride, and a review of a study analysed studies concerned with smart cloth that works by means of active cooling methods and a technology that works with lithium ion fibre batteries, it has proven its effectiveness in cooling the body by reducing the body temperature by less than the outside temperature, and the current study differed using nanopore polyethylene that is able to cool the body without sweating.

\section{Applied study procedures}

The current study aimed to present a proposal for designs for headscarves, with smart fabrics for women with Multiple sclerosis, by analysing and studying the most important aspects and principles of designing headscarves, in terms of: the functional aspect, the aesthetic aspect, and that is through field analysis of the products available in the market and comparing them with the analysis of the questionnaire to find out the needs and requirements of the sample.

\section{Outdoor clothing in Saudi Arabia:}

The women's abaya consisting of a headscarf and a coat is an essential external clothing in Saudi society, as it is worn by both Saudi and foreign women, and it is worth noting that Saudi women did not preserve any of the traditional clothes except for the abaya, which remained despite the changes that occurred to it from the single most important traditional dress in a woman's life (Al-Bassam, 1992).

\section{A field study of the (hijab) outdoor clothing available in the market:}

Currently, there are different styles of headscarf in some Saudi markets, as we find in the Saudi market the traditional abaya and the new abaya that are designed in the form of a coat (Tashkandi, 2014). And because of the strong correlation between headscarf and Abaya as an integrated costume in the Kingdom of Saudi Arabia, a previous questionnaire was used that strengthens the importance of headscarf for the sample. Where the sample consisted of 215 community women, and it shows the number of times an abaya was purchased during one year. The sample answered by purchasing 1-2 Abaya every year at a rate of 47\%. And this is what makes the study also pay attention to this aspect, which is that the abaya is a robe that is carefully selected in terms of its functional aspects so that it can be used on a daily basis, as confirmed by the sample of the study, which consisting of 77 women with Multiple sclerosis, that $35.1 \%$ of the sample go out from home on a daily basis for various reasons. Among them are going out for a job or going to hospital appointments, so the research has tended to analyse the design elements and its foundations in the Abaya available in the market, including:

\section{Functional and aesthetic component:}

There is a large number of designs for headwear suitable for the community in the local market, in terms of length and variety of models and colours, Where headscarf can perform their function, which is the element of coverage, as (Helali, 2005) defined the veil in general as everything that covers the head, such as a garment or other, and it may be called the shirt, the khimar, and the robe, and it is a wide garment for women that is wider than the robe, and by which women cover their head, chest and back. 
There are also a number of different materials for abaya and headscarf fabrics in the local markets that perform a number of different functions such as water permeability, heating, ease of wearing and care. Where the headwear and Abaya made of $100 \%$ polyester are perfect for wearing in hot climates. Likewise, linen, which is a fibre that is more durable than cotton, but it is a fabric that is easy to wrinkle and needs more care, unlike linen, we find elastic crepe fabrics that keep their lustre for a longer period without wrinkling, and Japanese fabrics are known now in the market for their softness and suitability in hot climates, and the ability to resist water and frizz more than other natural fabrics, and from the fabrics for headwear and Abaya, we find georgette, poly, and lightweight fabrics such as chiffon. These are the most prominent functional characteristics available for headscarf and Abaya in general in the local markets. (Al-Amari Textiles Stores).

\section{The element of modesty:}

The study sample answered that $49 \%$ of them prefer covering and modest headwear, and this item is available in the local markets, where you can find a large amount of headwear in bold colours and simple designs.

\section{The element of its suitability for special groups:}

Special categories refer to individuals who have differences and requirements from a natural person, (Adeelah, 2012) defined it as a term that denotes physical disabilities. As defined by the World Health Organization as follows: "Disability is a term that covers impairments, activity restrictions, and participation restrictions. A disability is a problem in the function or structure of the body, and therefore disability is a complex phenomenon, which reflects the interaction between the features of a person's body and the features of the society in which he or she lives." (World Health Organization, 1980). And through the field study of local markets, and as far as the researcher knows, she did not find many specialized fabrics for Abaya to reduce the negative effects of sunlight for the group of people with Multiple sclerosis, and through the search, we find fabrics called cooling towels, and in the study of (Wenfang, 2019), smart fabric is symbolized by WEC, which is made of lightweight nano-fabrics intended for athletes to reduce their body temperature, but one of the disadvantages of this material is that it needs to be soaked in water every 30-40 minutes to restore its cooling function, and this does not suit the study's orientation, as for the American Daily Health site, it has offered many materials and designs available for Multiple sclerosis patients to reduce the effects of heat from exposure to sunlight and other things, but the designs do not include ideas for covering the head. https://ara.dailydosehealth.com.

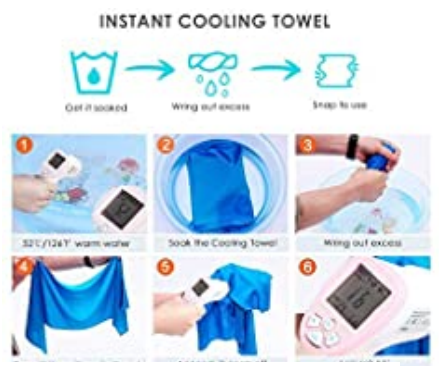

Picture 2 Cooling towel

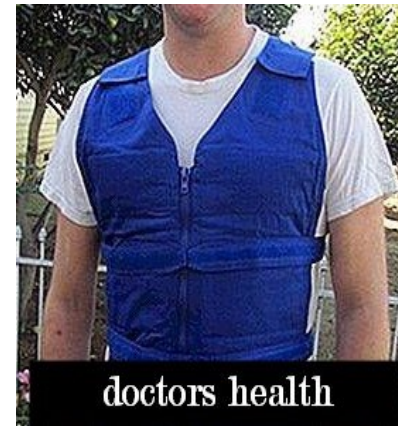

Picture 3 Cooling Vests for the Torso

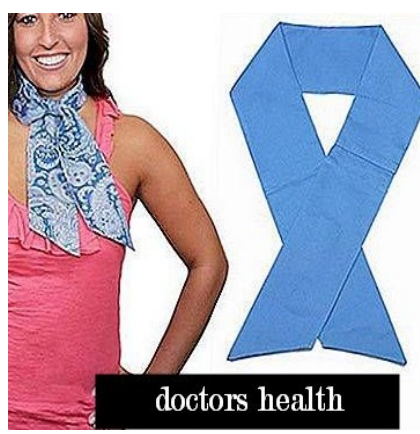

Picture 4 Heat-relieving shawl around the neck 


\section{Sample needs and requirements for designing a smart fabrics headscarf:}

Through a questionnaire directed to people with multiple sclerosis in the Kingdom of Saudi Arabia. And under the light of a number of axes, Results were reached that serve the goal of the research to reach the sample's needs for a headscarf that contributes to solving the problems of exposure to sunlight and high body temperature by proposing aesthetic designs for a suitable headscarf. * Appendix No. (1).

\section{A. General information about the sample:}

For 77 of people with multiple sclerosis, we find that the number of years of their diagnosis with the most frequent disease ranges between one to five years, where the duration of the incidence factor, with increased setbacks, influences external influences such as climate fluctuations faster (Khraifiye, 2015). In terms of age, the sample constituted $49.4 \%$ between $31-40$ years old, which is the target age in the study, the age of youth and activity, as for the climate, the sample answered, by $67.5 \%$, that it lives in hot summer and moderate winter areas in the Kingdom of Saudi Arabia.

\section{B. The functional requirement satisfying the hygienic comfort provided by the proposed} headscarf:

In terms of the functional requirements of the sample in designing a cover with smart fabrics, it contributes to solving the study problem:

The sample answered through the question / Do you feel that the sun's rays affect the development of symptoms of the disease. The sample confirmed yes by $71.4 \%$, which confirms the importance of the research, the sample also reported some of the most frequent development of symptoms (such as slurred speech, lethargy, impaired movement, blurred vision, headache). And the sample, by $68.8 \%$, indicated that it did not have solutions to reduce exposure to high temperature when exposed to sunlight, the sample called for covering the most affected organs, and the head represented the selection of the highest from the sample, followed by the back, the neck, and then the entire face.

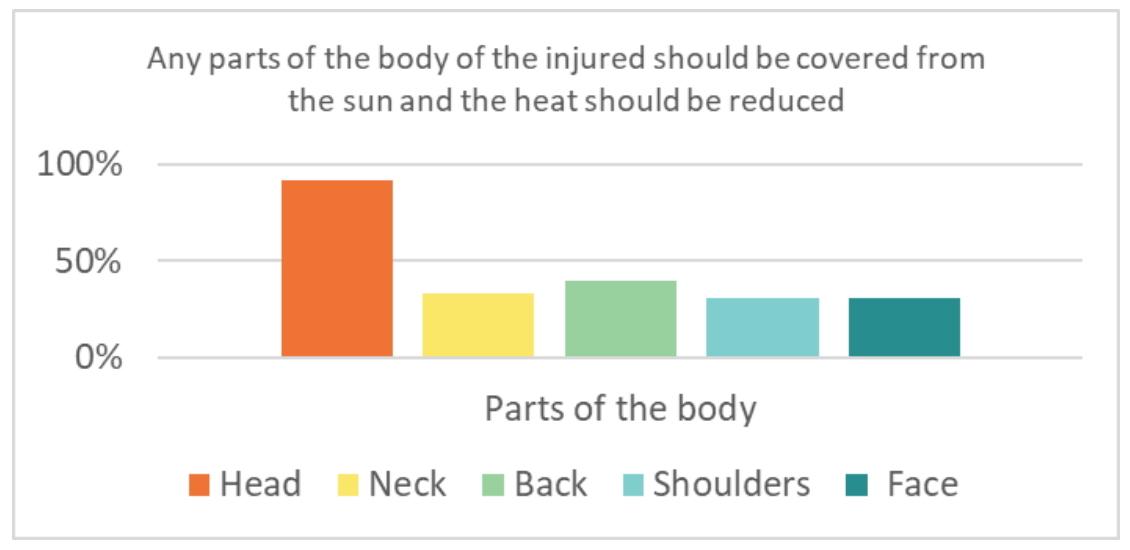

Figure 1: Results of the analysis of the questionnaire directed at female victims.

The sample also indicated its desire to use smart fabric, which contributes to solving the study problem with a vote rate of $69.2 \%$, and $49 \%$ of the sample asked for a decent headscarf, and the sample also wanted a comfortable and easy-to-use headscarf, by $50 \%$. 


\section{Requirement for aesthetics provided by the proposed headscarf:}

The sample focused on the modesty component of the shape of the outer covering, so the percentage of this aspect reached the highest percentage in the axis of the aesthetic requirements of this sample with a rate of $61.9 \%$, as for the outline of the designs, the sample focused on simple and uncomplicated designs that make wearing the cap practical. As for the lowest percentage, it was in favor of the aesthetic aspect, by $31 \%$, and this confirmed the greater percentage of the question if the sample would like to design a "veil" headscarf that shows the identity of the disease, as the sample answered no by $72.6 \%$. and the remainder of the sample voted yes if it was not in an exaggerated manner.

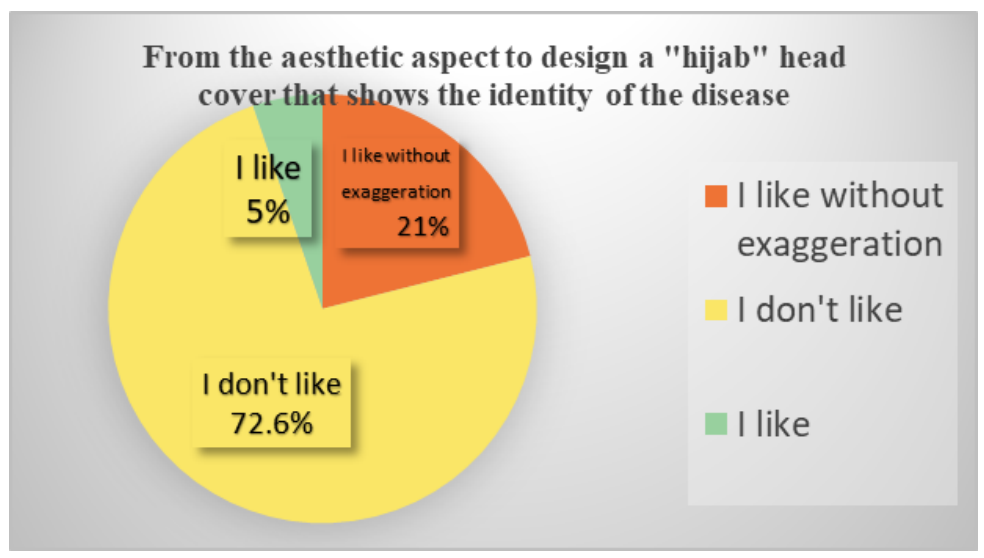

Figure 2: Results of a questionnaire analysis directed at affected women

\section{Suggested designs \\ Designs:}

Based on the sample's requirements and needs, three simple and easy-to-wear designs were created:

1. Bandana is worn under the regular headscarf.

2. A full headscarf that covers the neck and shoulders.

3. Scarf-like headscarf. 
Table 1 shows a description of the first proposed design

\begin{tabular}{|c|l|}
\hline Picture \\
design \\
Industrial \\
Front + eyes cover \\
the back to facilitate the wearing of the product by the sample, and the usual \\
hijab can be worn over the product, and made of smart fabric that helps cool the \\
head without sweating in case the head temperature rises due to exposure to hot \\
sunlight. The model has two shapes of a bandana without a cap that covers the \\
eye and a bandana that contains a cap that covers the eye according to the needs \\
of the sample. It can be available in any color as desired by the sample. \\
\hline Model colors light colors Carrying the disease awareness slogan
\end{tabular}

Table 2) This table shows a description of the proposed second design

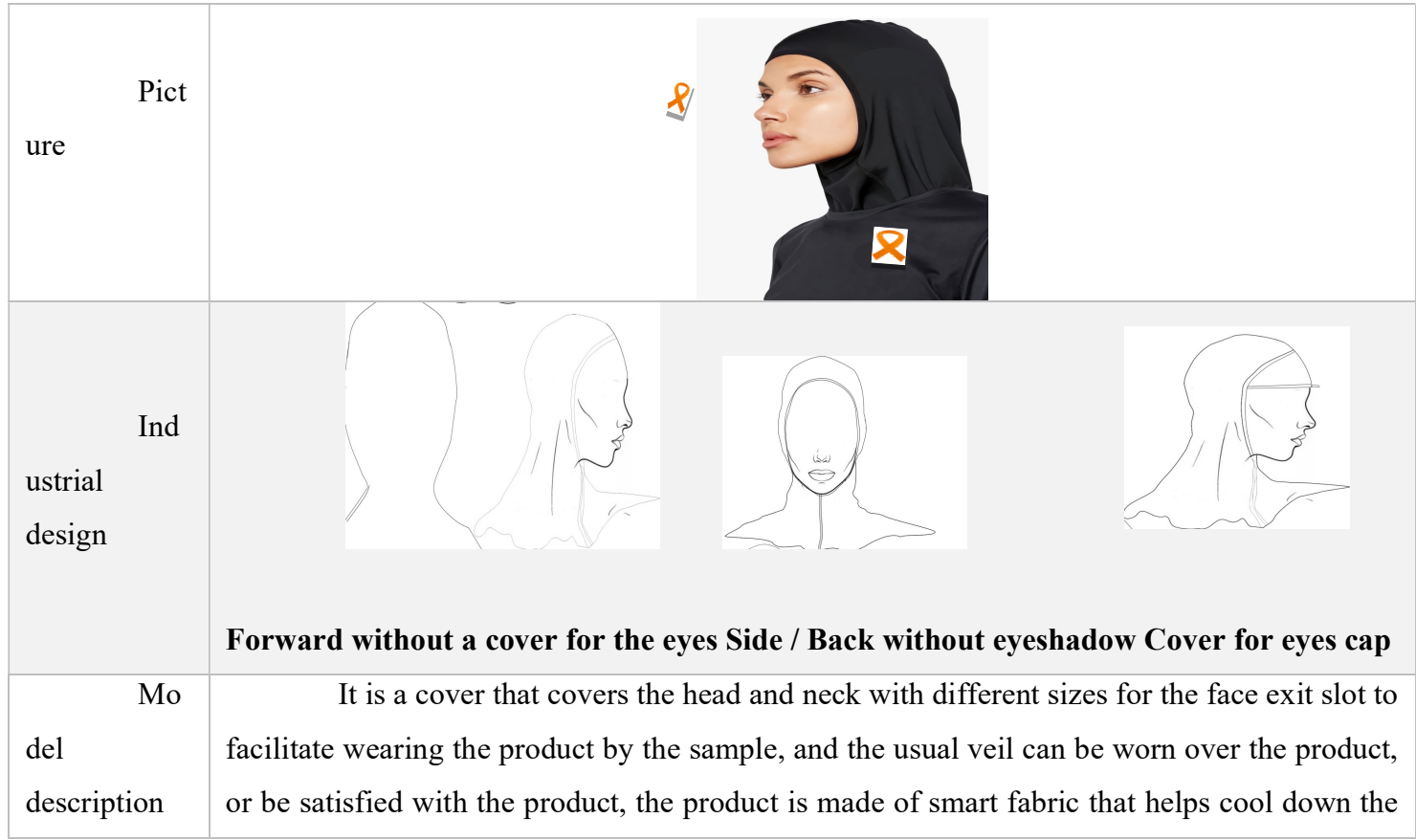




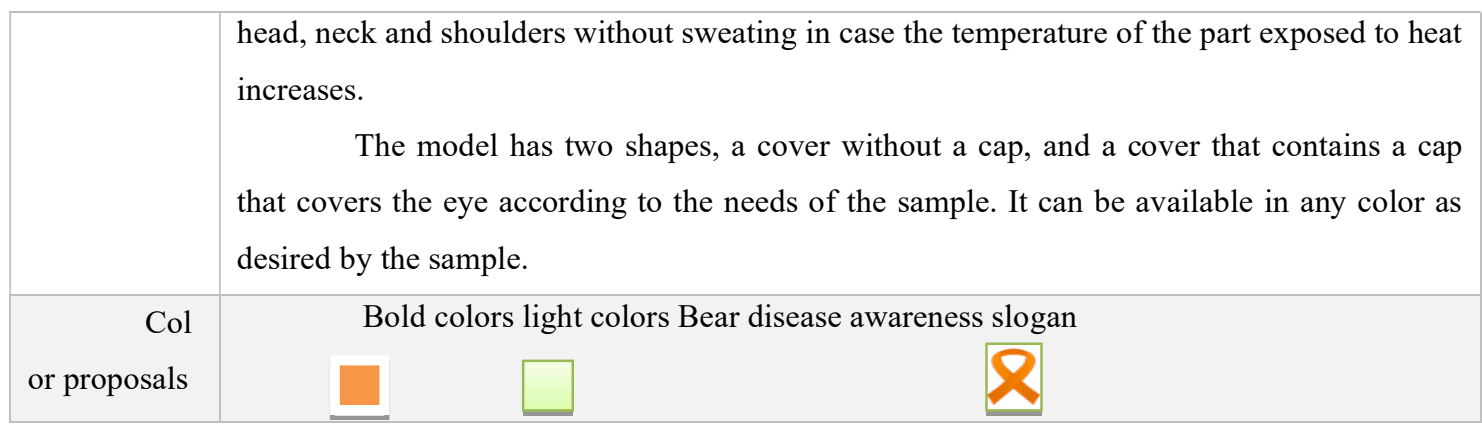

Table 3. The table shows a description of the third proposed design

\begin{tabular}{|c|c|}
\hline Pict & \\
\hline $\begin{array}{l}\text { ustrial } \\
\text { design }\end{array}$ & \\
\hline & $\begin{array}{l}\text { Forward without a cover for the eyes Side / without eyes cover } \\
\text { A cover (cap) to cover the eyes }\end{array}$ \\
\hline $\begin{array}{l}\text { Mo } \\
\text { del } \\
\text { description }\end{array}$ & $\begin{array}{l}\text { It is a cover that covers the head, neck, shoulders and back in different sizes, the } \\
\text { product is easy to wear by the sample, It replaces the usual wearing of the hijab, the product is } \\
\text { made of smart fabric that helps cool down the head, neck, shoulders and back without } \\
\text { sweating in the event that the temperature of the part exposed to heat increases. } \\
\text { The model has two shapes, a cover and a bandana without a cap, a cover and a band } \\
\text { that contains a cap that covers the eye according to the needs of the sample. }\end{array}$ \\
\hline $\begin{array}{r}\text { Col } \\
\text { or proposals }\end{array}$ & Bold colors light colors Bear disease awareness slogan \\
\hline
\end{tabular}

\section{Research tools}

The researcher built a questionnaire directed at women with Multiple sclerosis to measure the degree of their acceptance of the designs of headscarves, to protect them from exposure to the heat of sunlight. The questionnaire was divided into three axes:

1- General information axis and it included five questions that combine confirmation of infection, number of years of diagnosis, climate in which the infected person lives, marital status, and age.

2- $\quad$ The second axis measured the sample's views and perceptions about the smart cloth, through 4 questions that included an understanding of the definition of the smart material used in the designs, and 
choosing the most appropriate design through two sides, the first side includes the way the piece is worn under the abaya in a manner that is not visible to the public, and the other side includes the method of wearing a complete piece and appears to the public.

3- $\quad$ The questionnaire also included a measurement of the sample selection axis, for designs from several aspects, including the best color proposals for each design proposal, and the questionnaire tended to measure the functional aspect of the design, the functional aspect of the material, the aesthetics and the colors, and finally the principle of modesty through 11 questions that clearly measure their opinions based on attached pictures.

* Appendix No. (2).

\section{Research Results}

\section{1- General data of the sample}

The questionnaire was distributed to $77 \%$ of the infected sample. The sample varied in terms of the social level, as the percentage of female employees reached $43.8 \%$. As for non-employees, it was $56.3 \%$. The sample tended to a large extent for the sample that lives in areas with a hot summer and moderate winter climate. As for age, the most frequent age of the sample was between $31-40$ years, at $49.4 \%$. As for the number of years of diagnosis, the most frequent percentage of women diagnosed with Multiple sclerosis was from one to five years, with a rate of $56.3 \%$. Therefore, we conclude from general information the importance of the number of years of diagnosis, as the more years of diagnosis, the more sufferers are exposed to an increase in symptoms as a result of an external influence such as climate, we also conclude from this the relationship of the exacerbation of symptoms due to the female patients leaving for work, which is less than the middle of the sample, and this gives a clear indication that one of the reasons for the sample's unwillingness to go out to work may be due to climate-related reasons.

2- $\quad$ Results of the second axis (the functional aspect of the smart material idea and the outer lines of the design):

After the sample confirms the $100 \%$ understanding of the smart cloth's working mechanism and its use in the cover, we find that the sample tends to the first suggestion, which is to wear the cap as a bandana under the regular headscarf as a separate interior piece, and this requirement was compatible with the desire of the sample in the first questionnaire. As well as before seeing the proposed designs in the rest of the questionnaire. Where the sample chose the tendency for dark colours (such as black to Gray), and assures us the tendency of the sample to choose the first proposal is the period of diagnosis 1-5 years in which the percentage of symptoms aggravation decreases and the head for the sample is almost the only area that affects the increase in symptoms.

\section{3- $\quad$ Results of the third axis (functional - aesthetic aspect - the principle of modesty):}

Statistics show us a variety of opinions of the sample in the designs, and we may find the difference apparent due to the ability of the injured or not, to withstand the heat, and the real influencer on the questionnaire was the duration of diagnosis, the patient's age, as well as the marital status. Therefore, the third axis was divided into three items based on the requirements of the sample previously, and a picture was attached to the sample showing the differences between the three designs to facilitate selection on them. 


\section{- The first item: the functional aspect:}

The functional aspect fulfilled a number of requirements for the sample, including the final form after performance. Where the largest proportion of the third proposal came, which is the headscarf similar to the usual cover, followed by the second proposal and then the first. As for the most proposed aspect that contributes to the practical aspect in terms of ease of wearing and taking it off. We find that the sample tended to move towards the second design, followed equally by the rest of the two proposed designs. In view of the functional aspect that the design achieves in terms of (Which of the following designs do you find most contributing to solving your exposure to the sun's heat problem?), We find that the sample tends toward the second and third designs because it is the most covering of the head, neck and shoulders, which are the requirements and needs of the sample in the first questionnaire.

From the above, we find that the second design was the most functionally chosen.

\section{- The second item: the aesthetic aspect:}

This aspect measured the requirements and needs of the sample in terms of the most aesthetic design, and the appropriate colours to accept designs if they were put on the market, therefore, we find that the most aesthetic design from the sample's point of view was the second proposal at a rate of $56.3 \%$, followed by the third proposal, and then the first. As for the colours, the sample tended significantly for the third and first design proposal, to colour - black - Gray. As for the second design, which is the one that is more acceptable to the sample, opinions differed regarding the colours according to the ratio of approximation between the three colour proposals.

\section{Favorite colors}

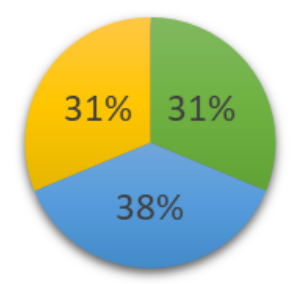

\section{Black to gray gradient}

Any colors are suitable

Material bearing designs for disease awareness logos

Figure 3: Preferred colors for the second design

\section{Third Clause: The Principle of Modesty:}

Through this item, the researcher reached the fulfilment of the sample requirement through the first questionnaire, in which the sample demanded the availability of the element of modesty at a rate of $61.9 \%$. Where the sample chose the third design as the most realization of this item, followed by the second design proposal and then the first. 


\section{Achieve the principle of respect.}

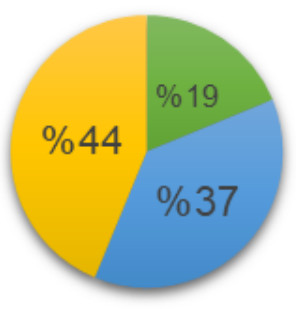

First proposal

The second proposal

\section{Third proposal}

Figure 4: The sample views on achieving the principle of modesty, in the three design proposals

\section{The most suitable designs for women with Multiple sclerosis:}

The researcher made designs for women with Multiple sclerosis that fit the needs and requirements previously analyzed in terms of a number of aspects, using a program:

\section{- $\quad$ First design proposal}

The first proposal did not achieve the highest percentages through the sample opinions, but less than a third of the sample preferred to wear such a proposal in black and without a cap covering the eyes.

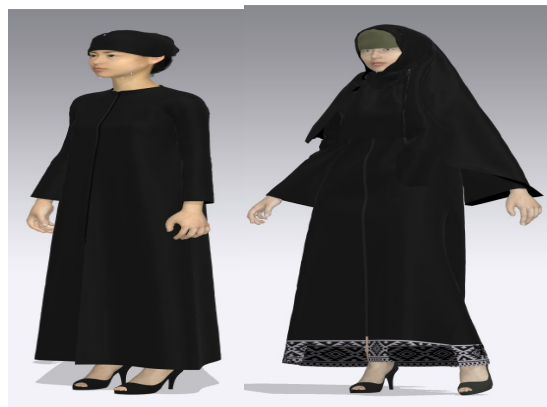

Figure 5: The first design proposal based on the sample request

- The second design proposal:

The second proposal achieved the highest proportions in terms of the sample's acceptance of the design, which achieved a number of functional aspects such as ease of wearing and taking off, and the sample was divided into a supporter of wearing a cap that is complementary or without a cap to protect the eyes from the sun, as well as achieving the highest aesthetic design, as the sample wanted a multicolored design. 

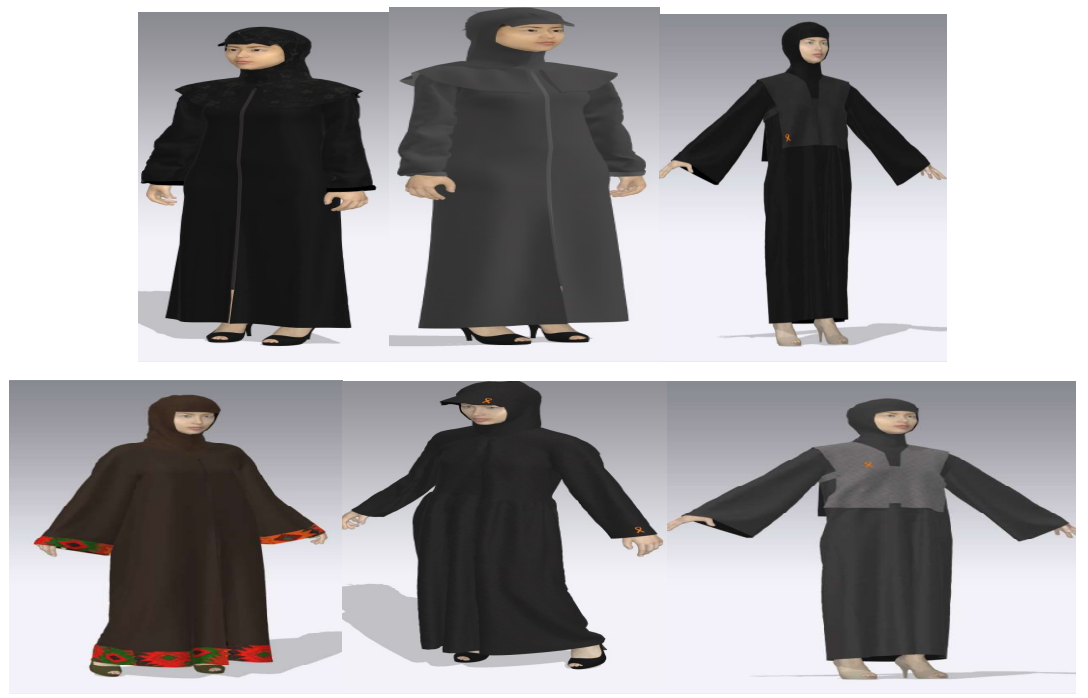

Figure 6: The second design proposal based on the sample request

\section{The third design proposal:}

The third proposal achieved the highest proportions in terms of the principle of modesty, and the sample preferred the black color for this type of designs, and the largest proportion of the sample did not want to put a protection for the eyes.

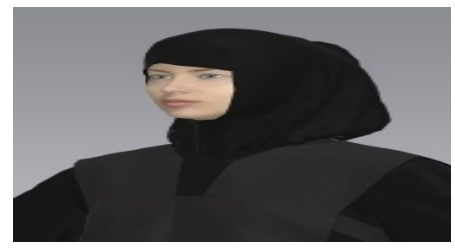

\section{Figure 7: The third design proposal based on the sample request}

\section{Conclusions}

Through the statistical analysis and technical comment of the sample data, the researcher summarized the results in several points, including:

- The number of years of diagnosis and marital status influenced the choice of the design most appropriate for the sample under study from a functional point of view of the smart tissue.

- We find a strong correlation between the design requirement that achieves modesty and the choice of appropriate colors for each type of proposed design.

- The sample was dominated by years of diagnosis in early stages of the disease, so we do not find interest in the sample to cover the eyes, and this came in proportions close to that of the sample diagnosed at one year to five years.

- $\quad$ The sample praised its great need for the material to help reduce heat without sweating, which contributes to improving the life of the sample.

- The combination of the requirements of the sample and its health needs contributed greatly to the arrival of a design proposal for the headscarf, "which is the second proposal". 


\section{Recommendations}

From the previous results, the researcher recommends the following:

- $\quad$ The health needs must be constantly examined when designing clothing proposals for the groups under study.

- $\quad$ Conducting more studies on designing clothes for special groups that contribute to improving life.

- Holding seminars and workshops that raise awareness among the public, in order to learn about the patients' dressing needs.

- $\quad$ Adopting the ideas in the research by experimenting with them and measuring the results, so that they can be produced and used.

\section{References}

Abyan, Lina Ahmed (2013) Genetic variation of alpha monocytes to the receptor of interleukin in Jordanian Multiple sclerosis patients: Master Thesis, The University of Jordan, pp. 1-82.

Adela, I. (2012) Self-concept and depression in persons with physical physical disabilities in the Bethlehem governorate. Published MA thesis: Al-Quds University.

Al-Ani, H. (2017) Sustainability of smart fabrics in indoor environment designs: Journal of the College of Education for Girls for Human Sciences, 11 (20).

Al-Bakri, A. (2010) Clothing and Human Health in the Twenty-first Century: Journal of Specific Education Research, 17 pp. 490-516.

Algahtani, Hussein Bader Shirah. Abdulrahman Bayazeed, Abdullah Alghamdi. Malik Almailabi, Mohammed Agharib (2020) Assessment of the Quality of Life of Multiple Sclerosis Patients' Caregivers in Saudi Arabia: Multiple Sclerosis and Related Disorders 37.

Al-Jaishi, H. (2012) Sustainability of smart fabrics in interior environment designs: Journal of the College of Education for Girls for Humanities, 11 (20).

Al-Nahas, R. (2014) Nanotechnology and the production of protective clothing for some groups at risk of UV rays: International Design Journal, 4 (4) pp. 223-215.

Antonia Kaltsatou, Andreas D. Flouris (2019) Impact of pre-cooling therapy on the physical performance and functional capacity of multiple sclerosis patients: A systematic review: Multiple Sclerosis and Related Disorders, 27 Pp 419-423.

Ashmawy, M. \& Madi, M. (2015) Problems of Manufacturing Outerwear for Women, Women's Abaya: Home Economics Journal, Helwan University.

Balcer, L. (2007) sclerosis multiple elapsing with Patients in loss visual reduces Natalizumab: Neurology 68 (16).

Bartkowiak, G., Dabrowska, A., \& Marszalek, A. (2017) Assessment of an active liquid cooling garment intended for use in a hot environment. Applied Ergonomics, 58, 182-189.

Chapman. A.R. (2013) Protection for Textiles Smart: Protection for Textiles Smart. P229.

Gad, M. (2010) The effect of methods used to produce polyethylene yarn on the mechanical properties of filter 
fabrics: Journal of Science and Arts - Studies and Research, 22 (4), pp. 95-108.

Ghazali, J. (2018), Cognitive Disorders in Multiple Sclerosis: Albaserah Center for Research, Consulting and Learning Services, 7 pp. 99-116.

Helli, A. (2005) The veil of the woman: the cultural rationale and the textual connotations: The Arab Journal of Human Rights, 11, pp. 21-33.

Jamal, M. (2004) Smart Garments, History, Technologies and the Future: Managing the Ready-to-Wear House System, The National Campaign for the Advancement of Textile Industries, Part Three, National Research Center.

Kharayefeh, A. (2015) The quality of life of patients with multiple sclerosis: Researcher Journal, 7 (13), pp. 148.

Lijun Lu a , Yajie Hu b , Kun Dai a (2020) The advance of fiber-shaped lithium ion batteries: Materials Today Energy. 5 p p 24-33.

Mattile. H. (2006) Clothing \&Textiles Intelligent: publishing Woodhead, p434

Muhammad, S. \& Syed, S. (2019) Smart Fashion Design: Arab Thought House.

Nga Nguyen, Thi-Thuy a, Ying-Hung Chen a , Min-Yi Chen a , Kuo-Bing Cheng b , Ju-Liang He a.(2017) Multifunctional Ti-O coatings on polyethylene terephthalate fabric produced by using roll-to-roll high power impulse magnetron sputtering system: Surface \& Coatings Technology, 324 Pp 249-256

Slimane, Hashim. Xue, Donga. Tao, Zhaoab (2020) Functionalization of polyethylene terephthalate knitted fabric with cowpea protein and biopolymer complex: Applications for enhancing wettability and UVProtection properties: Journal of Colloid and Interface Science 565 pp 367-360.

Song, N.M.- LeiJ.Leia, Z.-M. Lia. (2020) Spectrally selective polyvinylidene fluoride textile for passive human body cooling. Materials Today Energy.

Tashkandi, S. (2014). An investigation of thermal comfort properties of abaya under heat stress.

Thompson.al.et, (2000) Diagnostic criteriacal for peim progressive multiple sclerosis: Ann Neuol. Pp- 44-52.

WeiWeia1YongZhua1QiuLiaZefengChengaYongjiYaoaQiZhaoaPanZhangaXiaopengLiua (2020) An A12O3cellulose acetate-coated textile for human body cooling. Solar Energy Materials and Solar Cells.

Wenfang, Song. Faming, Wang. Chengjiao, Zhang (2019) Intermittent wetting clothing as a cooling strategy for body heat strain alleviation of vulnerable populations during a severe heatwave incident: Journal of Thermal Biology, 79. pp 33-41.

Yi Cui, Shanhui Fan, Po-Chun Hsu, Alex Y. Song, Peter B. Catrysse, Chong Liu, Yucan Peng, Jin Xie (2016) Radiative human body cooling by nanoporous polyethylene textile: $\underline{\text { science mag. }}$

\section{Websites}

(Saudi Ministry of Health. Multiple sclerosis statistics) https://www.moh.gov.sa/Search. https://laws.boe.gov.sa/BoeLaws/Laws/LawDetails/c99fc50a-eeb9-46ab-8144-a9ec01007992/1.

Al-Jishi, A. (2012), Tuesday, December 18, 2012 - 04 Safar 1434.

11,000 Gulf nationals have multiple sclerosis. Alwasat news.com. https://www.youtube.com/watch?v=bYdAXhLIL3Mhttps://ara.dailydosehealth.com 


\section{Appendix No(1) .}

Study title: Design proposals for a headscarf made of smart tissues for women with Multiple sclerosis in the Kingdom of Saudi Arabia

Multiple sclerosis is a chronic disease that affects young people and leads to partial or complete disability, God forbid, in the future, and we find an increase in the number of people with multiple sclerosis in the Gulf region, reaching 11,350 male and female injured (Al-Jishi, 2012), and based on what was stated in the initiatives of the National Transformation Program 2030 in the Kingdom of Saudi Arabia, where the Authority for the Care of Persons with Disabilities - which was established by a decision of the Council of Ministers in $1439 \mathrm{AH}$ - sought to increase the participation of people with Multiple sclerosis in the family and society, and to overcome the challenges facing them, in addition to harnessing all capabilities to serve them, and since the number of women affected by this disease is more than men, and based on the nature of the disease that focuses on the youth group, the research linked the problem of young Saudi women with this disease to their exit in the hot climates that prevail in most regions of the Kingdom, therefore, the aim of the research is to identify the ability of women with sclerosis to face the hot rays of the sun while going out for work or meeting personal needs during the day, by studying the proposed requirements for making designs for the "headscarf", as the research aims to reach proposals that contribute to solving the research problem in terms of function and aesthetics.

The survey is divided into three parts, where the survey begins with general information, then the second part of the survey, which identifies the ability of sclerosing patients to face the hot sunlight while going out, and the third part identifies problems and proposals for making a suitable headscarf for patients with sclerosis.

I ask you to participate by answering the questions contained in this questionnaire with accuracy, objectivity and clarity, which will only take you a few minutes.

Your participation is voluntary and will be confidential. All responses will be compiled and analyzed as a group.

If you have any questions or concerns, please contact the researcher / Doaa Khalaf Abdel Rahman. From the Department of Fashion and Textile, the clothing manufacturing course at King Abdul Aziz University in Jeddah - College of Human Sciences and Design. To the email: dabdurahmanalmalki@stu.kau.edu.sa.

I also thank you for your efforts, may God bless you with health and wellness.

\section{The first axis:}

\section{A. General information:}

This axis contains 5 questions

1. Health status:

* A patient with multiple sclerosis.

\section{How many years ago were you diagnosed with multiple sclerosis:}


* Open answer

\section{Marital Status:}

* Employee.

* Unemployed.

4. Age:

* Less than 20.

* 20-30

*30-40

*40 and more.

5. Climate condition:

* I live in a hot environment in summer and moderate in winter.

* I live in a hot environment all year long.

* I live in a climate volatile environment.

* I live in a cold environment in summer and winter.

The second axis: identifying the ability of sclerosing patients to face hot sunlight while going out for work or meeting personal needs during the day:

This axis contains 4 questions

1.How many times per week in your normal life do you have to go out during the day in hot weather?

* Daily

* Less than two days

* Two to four days

* I rarely go out of the house

2. Do you feel that the sun's rays affect the development of your disease symptoms if you are under the sun on a hot and sunny day?

* yes

* Example

* NO

* Sometimes

3. Do you have simple solutions to avoid sunlight While out of the house on a hot and sunny day?

* yes- Example

*No

4. Is covering the head from the sun's rays sufficient to reduce the effect of heat while leaving the house on a hot and sunny day?

* yes

*No

5. What are the organs affected by the heat as well? 
The third axis: proposals for making a suitable headscarf for patients with sclerosis:

This axis contains 4 questions

1. Is your current headscarf suitable in terms of its design and provides you with protection from the sun's rays?

* yes

*No

2. Would you like to have a headscarf that helps reduce heat through smart fabric while leaving the house on a hot and sunny day?

* yes

*No

3. What organs of the patient's body should be covered when proposing an intelligent and aesthetic design that contributes to solving the research problem, can more than one answer be chosen?

* Head

* Shoulders

* Full face

* The face, but in a way that contributes to vision

* Arms and hands

* Back

4. Do you want to design a "veil" headscarf that shows the identity of the disease?

* I want

* I do not want

* Desire not too much.

Thank you (-) 


\section{Appendix No(2) .}

Study title: Design proposals for a headscarf made of smart tissues for women with Multiple sclerosis in the Kingdom of Saudi Arabia

Multiple sclerosis is a chronic disease that affects young people and leads to partial or complete disability, God forbid, in the future, and we find an increase in the number of people with multiple sclerosis in the Gulf region, reaching 11,350 male and female injured (Al-Jishi, 2012), and It is a disease that affects the youth group, with a higher percentage of women than men, and since exposure to the sun's heat increases the symptoms of the disease, and it is the climate that dominates the regions of the Kingdom of Saudi Arabia, The aim of the research is to design proposals for a "hijab" headscarf made of smart fabrics (a nanofabric that cools the head of the injured without the need to soak the cap in water before wearing it), and this questionnaire seeks to reach proposals for a headscarf that suits the opinions and desires of the sample.

I ask you to participate by answering the questions contained in this questionnaire with accuracy, objectivity and clarity, which will only take you a few minutes.

Your participation is voluntary and will be confidential. All responses will be compiled and analyzed as a group.

If you have any questions or concerns, please contact the researcher / Doaa Khalaf Abdel Rahman. From the Department of Fashion and Textile, the clothing manufacturing course at King Abdul Aziz University in Jeddah - College of Human Sciences and Design. To the email: dabdurahmanalmalki@stu.kau.edu.sa.

I also thank you for your efforts, may God bless you with health and wellness.

\section{B. General information:}

This axis contains 5 questions

\section{Health status:}

* A patient with multiple sclerosis.

\section{How many years ago were you diagnosed with multiple sclerosis:}

* Open answer

\section{Marital Status:}

* Employee.

* Unemployed.

4. Age:

* Less than 20.

* 20-30

* 30-40

*40 and more.

\section{Climate condition:}

* I live in a hot environment in summer and moderate in winter.

* I live in a hot environment all year long. 
* I live in a climate volatile environment.

* I live in a cold environment in summer and winter.

The second axis: opinions and perceptions of the sample on the smart cloth:

This axis contains 4 questions

1. Through the proposal to design a headscarf made of smart cloth, what is the idea of smart cloth?

The smart cloth used in the proposed cover is a cloth that contains nanoparticles that contribute to reducing the temperature of the head in the event of exposure to external heat such as sunlight without the need to soak the cloth in water to obtain cooling, as it does not wait for the head to reach the stage of sweating to start cooling, it works on Self-cooling.

* Click to confirm understanding of the concept from the smart cloth

2. Do you want to wear the suggested design under the regular head scarf as a separate interior piece (similar to the men's hat)?

$*$ Yes

*No

3. In case you answered yes, and I want your headscarf to be one piece of smart fabric to wear under your usual headscarf - what colors are right for you?

*Dark colors

*Light colors

* Contains the Multiple Sclerosis Awareness Logo

4. In case you answered no, and you want your headscarf to be a single piece of smart fabric as a substitute for your usual headscarf - what are the right colors for you?

*Dark colors

*Light colors

* Contains the Multiple Sclerosis Awareness Logo

\section{The third axis: choosing design requirements:}

\section{This axis contains 11 questions}

1. Which of the following styles do Abayayou prefer in terms of wearing?

2. Which of the following styles do you prefer for practicality and ease of wearing and taking off?

3. Which of the following designs do you find achieve the principle of modesty for you?

4. Which of the following designs do you find most contributing to solving your exposure to the sun's heat?

1. Can you decide which colors do you prefer for Design No. 1 ?

1- Black-gray gradation

2- $\quad$ Any colors are suitable

3- Material bearing designs of disease awareness logos 
2. Can you decide which colors do you prefer for Design No. 2?

1- Black-gray gradation

2- $\quad$ Any colors are suitable

3- Material bearing designs of disease awareness logos

3. Can you decide which colors do you prefer for Design No. 3?

1- Black-gray gradation

2- $\quad$ Any colors are suitable

3- $\quad$ Material bearing designs of disease awareness logos

4. Do you prefer to wear an eye protection cap in Design No. 1?

5. Do you prefer to wear an eye protection cap in Design No. 2?

6. Do you prefer to wear an eye protection cap in Design No. 3?

Thank you 